\title{
Mastoid Vibration Reduces Ipsilesional Shift of Subjective Visual Horizontal in Patients with Acute Stage of Unilateral Vestibulopathy
}

\author{
Kun Woo Kim, Min Young Lee, and Jae Yun Jung \\ Department of Otorhinolaryngology-Head and Neck Surgery, College of Medicine, Dankook University, Cheonan, Korea
}

\footnotetext{
Received November 9, 2016

Revised December 29, 2016

Accepted January 9, 2017
}

Background and Objectives: To investigate effect of the vibration on subjective visual horizontal (SVH) in patients with acute stage of unilateral vestibulopathy. Subjects and Methods: Twenty-five unilateral vestibulopathy patients which analyzed into 42 cases at different time points and suffered from spinning vertigo for more than 24 hours without hearing loss and neurologic abnormality were enrolled. Thirteen subjects with spontaneous nystagmus ( $>3$ degree/sec; averaged symptom onset $<1$ week) at the time of SVH measurement were classified into the acute unilateral vestibulopathy group (aVU). The other 29 subjects without spontaneous nystagmus were classified into the compensated vestibulopathy group (cVU). SVH was performed with vibration at either mastoid or sterocleidomastoid muscle. Results: In the analysis of overall subjects, vibration did not significantly change the degree of shift of SVH. However, analyzed by group, the shift of SVH with vibration at ipsilesional mastoid was significantly decreased than baseline in aVU $(p<0.05)$. The shift of SVH with vibration at contralesional mastoid was significantly increased than baseline in cVU $(p=0.05)$. Conclusions: The shift of SVH due to vibration in acute stage of unilateral vestibulopathy showed reduction of the shift, while in compensated stage it showed increase of the shift.

J Audiol Otol 2017;21(2):66-71

KEY WORDS: Subjective visual horizontal · Vibration · Unilateral vestibulopathy · Shift Vestibular system.

\section{Introduction}

Visual orientation is provided by delicate integration of three major information which are derived from vision, vestibular organs, and proprioception. The loss of balance between two vestibular systems of each ear results in distortion of visual orientation, which could be assessed by subjective visual horizontal (SVH). Since Friedmann [1] introduced clinical application of subjective visual vertical (SVV) in peripheral vestibulopathy patients, SVV and SVH have been used in many dizziness clinics because of the simple test procedure and inexpensive equipment needed. Several studies reported that SVV deviates toward the ipsilesional side in vestibulopathy

This is an Open Access article distributed under the terms of the Creative Commons Attribution Non-Commercial License (http://creativecommons.org/licenses/by-nc/4.0/) which permits unrestricted non-commercial use, distribution, and reproduction in any medium, provided the original work is properly cited patients, and normal variation of the SVV has been reported within 1 to 3 degree [1-3]. The normal variation of the SVH also reported within 2 to 3 degree [4,5]. Moreover, Liu, et al. [4] reported that unilateral vestibular neuritis patients showed statistically significant improvement of SVH, SVV after rehabilitation.

However decreased shift after compensation has put a limitation on SVH for clinical usage for detecting vestibular imbalance at any given states. Karlberg, et al. [6] showed vibration method partly overcome this limitation in chronic unilateral vestibular deficits and suggested vibration can improve sensitivity of test. They showed applying vibration to mastoid bone and sternocleidomastoid muscle (SCM) on either side increased SVH shift to lesion side with greatest shift on ipsilesional SCM (IS). They explained this phenomenon with a theory of direct vibratory stimulation of intact vestibular receptors. But, we have observed some patients who showed less shift 
with vibration compared to baseline value in acute stage of unilateral vestibulopathy.

As far as we know, vibration on SVH during acute stage of unilateral vestibulopathy has not been reported yet. Therefore, we measured SVH to investigate effect of the vibration on SVH in patients with unilateral peripheral vestibulopathy in both the acute and compensated stage.

\section{Subjects and Methods}

\section{Patients}

Twenty five unilateral vestibulopathy patients (19 rightsided and 6 left-sided) who visited our clinic from 2009 to 2010 with a result of SVH test with vibration were enrolled. The 25 patients displayed typical peripheral vestibular weakness both in clinical manifestations and vestibular function test including SVH with vibration. Twenty-three patients had vestibular neuritis, one patient had iatrogenic vestibular weakness after surgery for vestibular schwannoma, and one patient had temporal bone fracture induced vestibular hypofunction. The 15 men and 10 women had a mean age of 47.6 (range, $32-70$ ) years. They all complained of spinning vertigo for more than 24 hours without hearing loss or neurologic abnormality.

In several patients we were able to measure spontaneous nystagmus and SVH with vibration at different time sequences during the compensation process. We considered data at different time points in the same patient as separate cases. Thus, we obtained 42 cases for analysis. Thirteen cases with spontaneous nystagmus ( $>3$ degree/sec; averaged symptom onset $<1$ week) at the time of SVH measurement were classified in the acute unilateral vestibulopathy group (aVU). The other 29 cases without spontaneous ( $<3$ degree/sec; averaged symptom onset $>1$ week) nystagmus were classified in the compensated vestibulopathy group (cVU).

\section{Vibration stimulus}

We used a hand-held vibrator (VVIB 100; Synapsys, Marseille, France) with a fixed frequency of $100 \mathrm{~Hz}$, an amplitude of $0.1 \mathrm{~mm}$, and a round contact surface (diameter 2.0 $\mathrm{cm}$ ). Two anatomical sites were vibrated, the mastoid bone, and neck muscle. The mastoid bone behind the external ear canal was vibrated and vibrator was held in position by hand. For vibrations of the neck muscles, SCM was chosen based on previous report $[6,7]$. To standardize the location of SCM vibration, the belly of the SCM and the mastoid bone were palpated during active muscle contraction, and a point on the muscle belly $50 \mathrm{~mm}$ below the tip of the mastoid bone was vibrated [ipsilesional mastoid (IM); contralesional mastoid (CM);

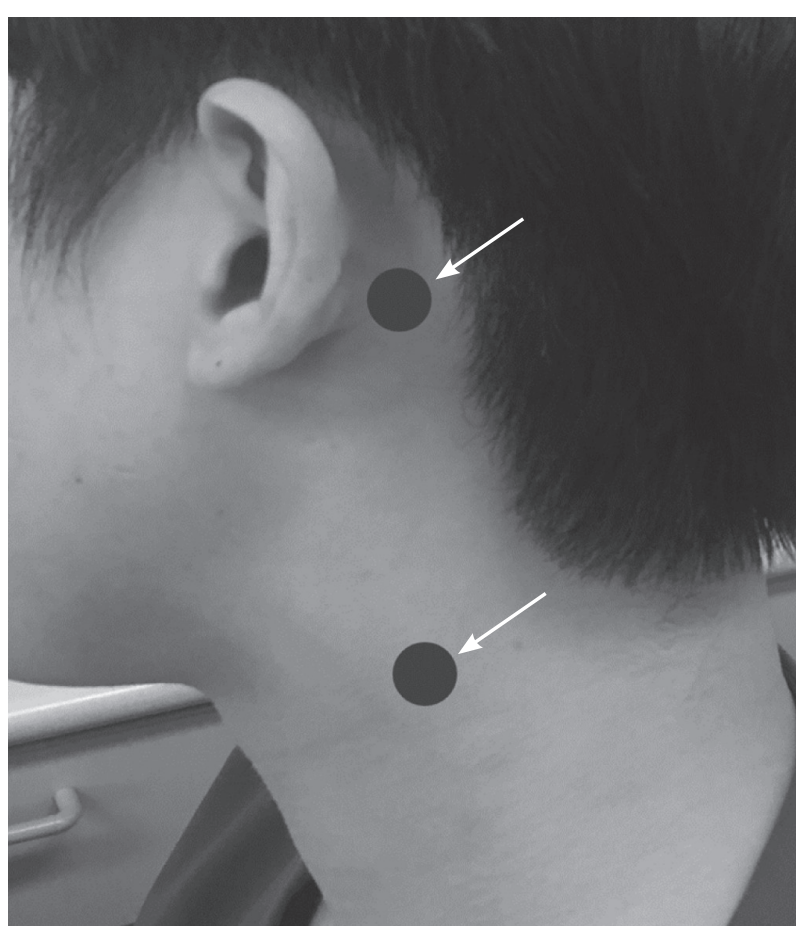

Fig. 1. Four different locations for vibrator applied during SVH test done on both sides of the mastoid area and the midline of both SCMs (arrows). SVH: subjective visual horizontal, SCM: sternocleidomastoid muscle.

IS; contralesional SCM (CS)] (Fig. 1) [6].

\section{SVH measurement}

Patients were seated in a dark room with upright position without head rest and asked to set light illuminating bar (80 $\mathrm{cm}$ long, $2 \mathrm{~cm}$ wide) in front of patients at a distance of 1.5 meters. The neck rest could be adjusted in the vertical and anterior-posterior directions to fit every subject. Control kit was prepared for patients to rotate the bar in a roll plane until they set the subjective horizontal. Baseline SVH measurement was done twice for each preset angle (clockwise $45^{\circ}$, counterclockwise $45^{\circ}$ ). Then, each patient was performed the SVH test with vibration applied both mastoid and SCM. Mean value of each test condition ( 4 values for baseline, 2 values for vibration test) was used for analysis. There was no time limit for finishing each test, so that patients took enough time for setting visual horizontal with confidence.

\section{Statistical analysis}

Statistical analysis was performed using SPSS version 18.0 for Windows (PASW Statistics; SPSS Inc., Chicago, IL, USA) and $p$ value of $<0.05$ was considered statistically significant. The results were expressed in means \pm standard errors. A Wilcoxon signed rank test was applied to compare the groups. 


\section{Results}

\section{Part I. Overall analysis}

Only 23 cases had caloric tests performed on the same day as the SVH and 4 cases were excluded due to inconsistency between the lesion side and canal paresis side. The canal paresis were $66.1 \pm 29.0,32.2 \pm 50.0 \%$ in the aVU group and $\mathrm{cVU}$ group, respectively. However, the canal paresis was not significantly correlated with the shift of SVH (Pearson's correlation coefficient $=0.351, p=0.140)$. Almost all $(41 / 42 ; 98 \%)$ of the patients had lesion side shift, with one patient (2\%) displaying contralesional shift. In the analysis of overall subjects (including both aVU and cVU), vibration did not significantly change the degree of shift of SVH. Without vibration it was $4.05 \pm 3.63^{\circ}$ and with vibration at IM and IS it was $3.53 \pm$ $2.94^{\circ}(p=0.110)$ and $4.49 \pm 3.42^{\circ}(p=0.150)$, respectively; the difference between the two was -0.52 and $+0.44^{\circ}$. Also, without vibration it was $4.05 \pm 3.63^{\circ}$ and with vibration at $\mathrm{CM}$ and CS it was $4.32 \pm 3.67^{\circ}(p=0.286)$ and $4.13 \pm 3.91^{\circ}$, respectively ( $p=0.733$ ); the difference between the two was +0.27 and $+0.08^{\circ}$ (Table 1). There was no significant change according to vibration location and side. These results suggest that vibration of both side and either location do not provoke the hidden vestibular dysfunction or increase the lesion shift (Fig. 2).

\section{Part II. Separate group analysis (aVU vs. cVU)}

In the analysis of aVU (with spontaneous nystagmus), without vibration it was $8.01 \pm 4.05^{\circ}$ and with vibration at IM and IS it was $6.24 \pm 2.47^{\circ}$ and $7.68 \pm 3.60^{\circ}$, respectively, and difference between the two was -1.77 and -0.33 . Without vibration it was $8.01 \pm 4.05^{\circ}$ and with vibration at CM and CS it was $7.49 \pm 3.96^{\circ}$ and $8.17 \pm 4.12^{\circ}$, respectively, and difference between the two was -0.52 and +0.16 in the aVU group (Table 1). The shift of SVH with vibration at IM was significantly decreased compared to baseline in the aVU group $(p=0.009)$. The shift of SVH with vibration at IS and CM showed decreasing tendency after vibration, but no significant differences were found at those locations in the aVU group ( $p=0.358$ and 0.197$)$. There were no case that the direction of nystagmus changed after the vibration.

As to the results from $\mathrm{cVU}$ (no spontaneous nystagmus), without vibration it was $2.28 \pm 1.34^{\circ}$ and with vibration at IM

Table 1. Baseline and vibaration-induced shift of SVH in group ( $p$-value)

\begin{tabular}{|c|c|c|c|c|c|}
\hline \multirow{2}{*}{ Groups } & \multirow{2}{*}{ Baseline } & \multicolumn{2}{|c|}{ Ipsilesional side } & \multicolumn{2}{|c|}{ Contralesional side } \\
\hline & & Mastoid & SCM & Mastoid & SCM \\
\hline Overall $(n=42)$ & $4.05 \pm 3.63^{\circ}$ & $3.53 \pm 2.94^{\circ}(0.11)$ & $4.49 \pm 3.42^{\circ}(0.15)$ & $4.32 \pm 3.67^{\circ}(0.29)$ & $4.13 \pm 3.91^{\circ}(0.73)$ \\
\hline Acute stage $+S N(n=13)$ & $8.01 \pm 4.05^{\circ}$ & $6.24 \pm 2.47^{\circ}(0.01)$ & $7.68 \pm 3.60^{\circ}(0.36)$ & $7.49 \pm 3.96^{\circ}(0.20)$ & $8.17 \pm 4.12^{\circ}(0.67)$ \\
\hline Compensative stage-SN $(n=29)$ & $2.28 \pm 1.34^{\circ}$ & $2.32 \pm 2.26^{\circ}(0.90)$ & $3.05 \pm 2.17^{\circ}(0.06)$ & $2.90 \pm 2.50^{\circ}(0.05)$ & $2.32 \pm 2.03^{\circ}(0.88)$ \\
\hline
\end{tabular}

Data are presented as mean \pm SD. SVH: subjective visual horizontal, SN: spontaneous nystagmus, SCM: sternocleidomastoid muscle

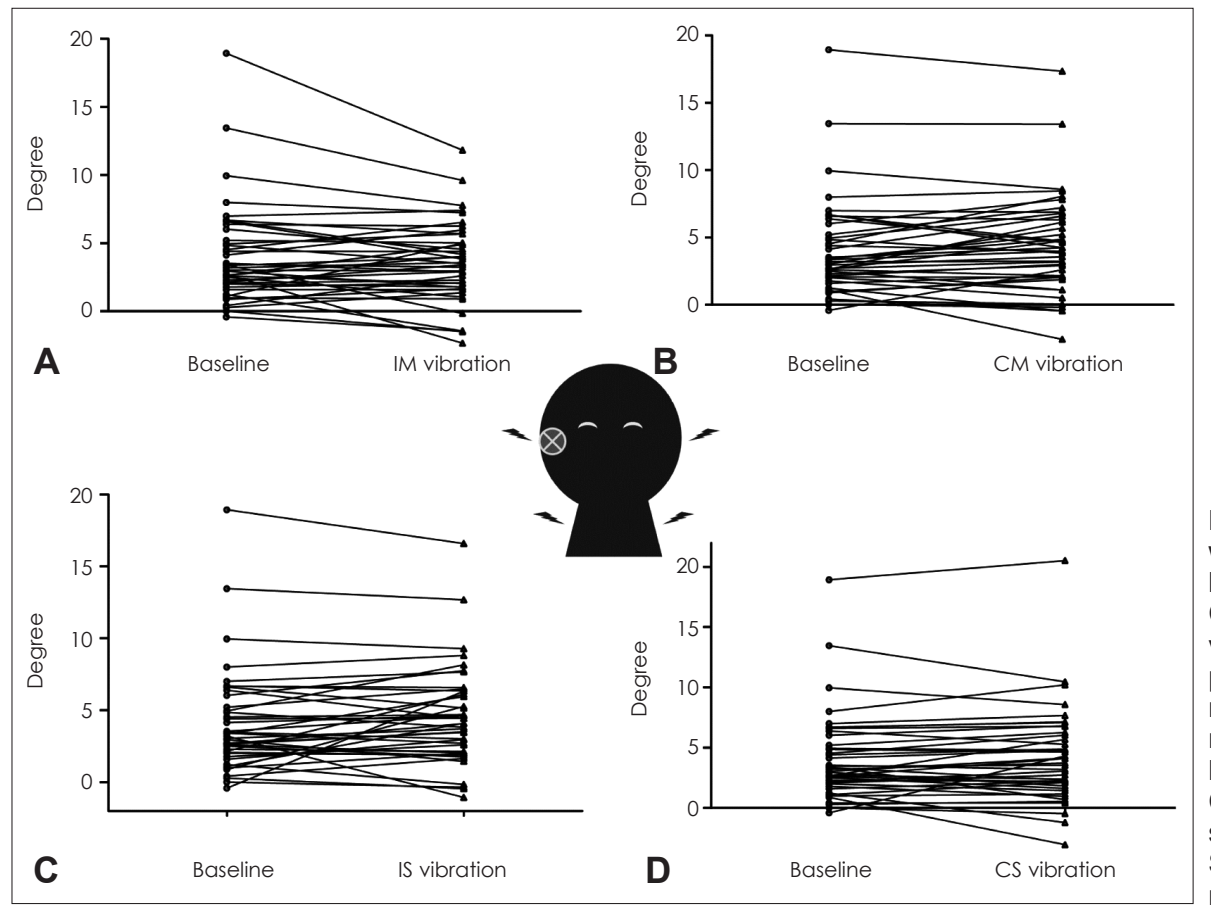

Fig. 2. The change of shift of SVH with vibration in unilateral vestibulopathy patients. A: IM vibration, B: CM vibration, C: IS vibration, D: CS vibration. These data include both patients with spontaneous nystagmus and those without spontaneous nystagmus. SVH: subjective visual horizontal, IM: ipsilesional mastoid, CM: contralesional mastoid, IS: ipsilesional SCM, CS: contralesional SCM, SCM: sternocleidomastoid muscle. 
and IS it was $2.32 \pm 2.26^{\circ}$ and $3.05 \pm 2.17^{\circ}$, respectively, and difference between the two was +0.04 and +0.77 . Also, without vibration it was $2.28 \pm 1.34^{\circ}$ and with vibration at $\mathrm{CM}$ and CS it was $2.90 \pm 2.50^{\circ}$ and $2.32 \pm 2.03^{\circ}$, respectively, and difference between the two was +0.62 and +0.04 in the cVU group (Table 1). The shift of SVH with vibration at CM was significantly increased compared to baseline in cVU ( $p=$ 0.049) (Fig. 3). The shift of SVH with vibration at IS showed increasing tendency after vibration, with a marginal level of significance $(p=0.056)$.

Vibration at IM displayed a significant reduction of the shift of SVH in aVU. On the other hand, vibration at IS and CM showed significant or near-significant increase of the shift of SVH in cVU (Fig. 4A). The shift of SVH was decreased by up to 7 degree (Fig. 4B).

In this study, there was a representative case changed from aVU group to cVU group. In the aVU (with spontaneous nystagmus), without vibration it was $6.59^{\circ}$ and with vibration at IM and IS it was $3.84^{\circ}$ and $5.15^{\circ}$, respectively. Also, with vibration at $\mathrm{CM}$ and $\mathrm{CS}$ it was $6.3^{\circ}$ and $6.76^{\circ}$, respectively. In the cVU, without vibration it was $2.07^{\circ}$ and with vibration at IM and IS it was 2.28 and $1.9^{\circ}$, respectively. Also, with vibration at $\mathrm{CM}$ and $\mathrm{CS}$ it was 2.73 and $2.06^{\circ}$, respectively. In the aVU, the shift of SVH showed decreasing tendency after vibration, and the vibration at IM was the most decreased. In the cVU, the shift of SVH showed increasing tendency after vibration, and the vibration at $\mathrm{CM}$ was the most increased (Fig. 5).

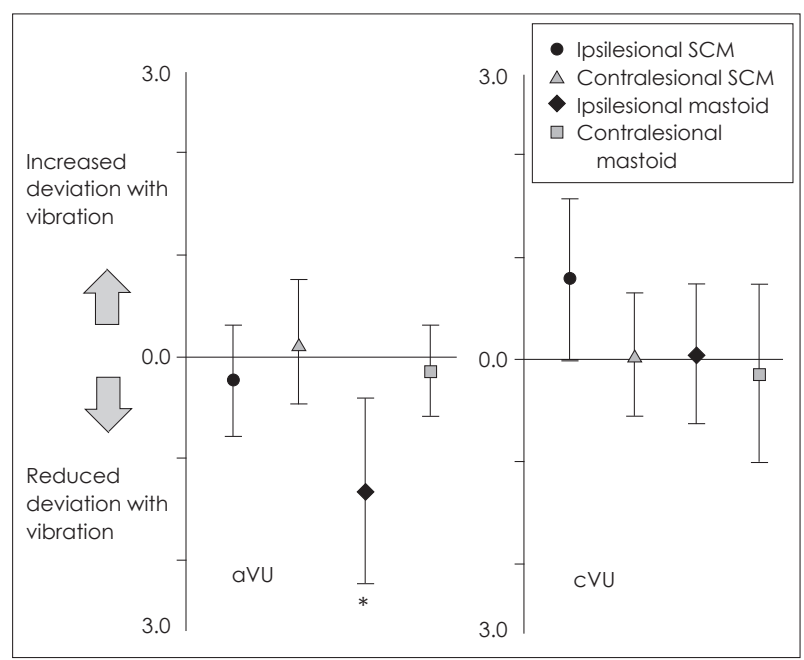

Fig. 3. Average difference between baseline shift of SVH and shift of SVH with each type vibration. Positive value means increased lesion side shift when tested and negative value means reduced lesion side shift. Vibration at in aVU and vibration at $\mathrm{CM}$ in cVU showed significantly changed. ${ }^{*} p<0.05$. SVH: subjective visual horizontal, aVU: acute unilateral vestibulopathy group, cVU: compensated vestibulopathy group, SCM: sternocleidomastoid muscle, CM: contralesional mastoid.
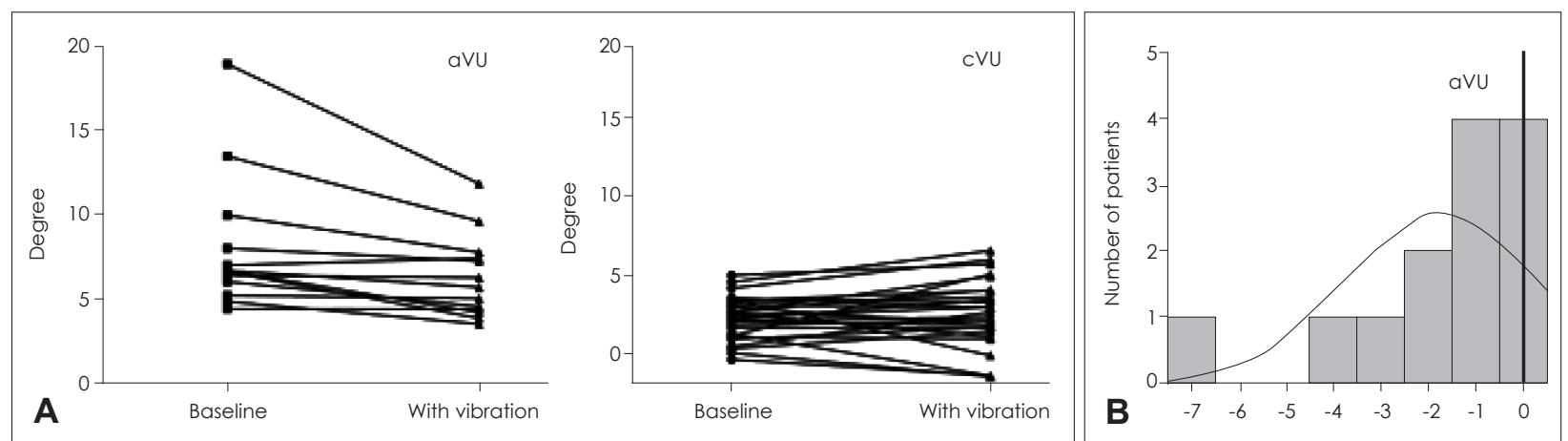

Fig. 4. The shift of SVH with IM bone in separate group. During the vibration at IM, the shift of SVH in aVU tend to decrease, however the one in CVU showed a variety of results but mostly increasing (A). Histogram of the SVH difference value between baseline and SVH with vibration at IM in aVU (B). SVH: subjective visual horizontal, aVU: acute unilateral vestibulopathy group, cVU: compensated vestibulopathy group, IM: ipsilesional mastoid.

Fig. 5. Representative case shows that vibration decreased SVH deviation in acute stage, but increased SVH deviation after compensation. SVH: subjective visual horizontal, aVU: acute unilateral vestibulopathy group, cVU: compensated vestibulopathy group, IM: ipsilesional mastoid, IS: ipsilesional SCM, CM: contralesional mastoid, CS: contralesional SCM, SCM: sternocleidomastoid muscle.
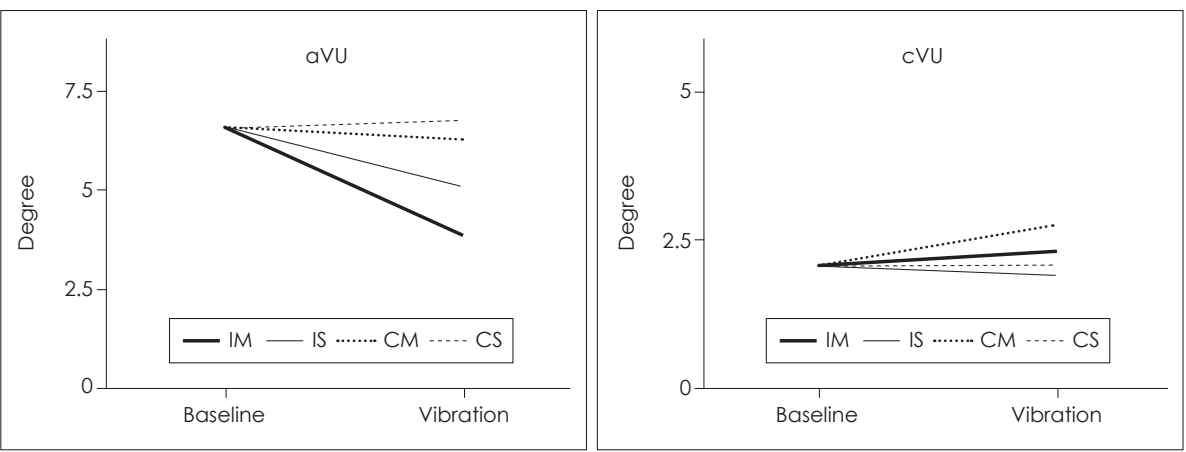


\section{Discussion}

Vibration can stimulate bilateral labyrinth and activate vestibular receptors [8]. It has been reported that vibratory stimulus of the head and neck could induce nystagmus in patients with vestibular disorders while it could not induce nystagmus in normal people [9]. The asymmetric signals derived from the unilateral vestibulopathy affect the midbrain and evoke distinguishing ocular movements, such as vibrationinduced nystagmus (VIN). Vibration with frequencies above $80 \mathrm{~Hz}$ delivered to the heads of squirrel monkeys was reported to excite the semicircular canal (SCC) and otolith afferents [8]. Another study showed that air-conduction sound activate vestibular receptors of otoliths, mostly macula of saccule [10]. Curthoys, et al. [11] showed that low-intensity $500 \mathrm{~Hz}$ boneconducted vibration activate a high proportion of otolith irregular neurons from the utricular and saccular maculae but few SCC neurons. Therefore, when the vibration directly stimulates the vestibular system, the responsible organ is regarded as otolith organs.

Vibration activates the vestibular system and the proprioceptive system of the neck, especially primary muscle spindle afferents. As vibratory stimulation to the neck muscle can stimulate the proprioceptive system, ocular movement like VIN might be evoked by a signal from the proprioceptors of the neck muscle [9]. The neck afferent signals provide information of head position, and the cervical proprioceptive system is especially important for body orientation [12,13]. Several studies have demonstrated that stimulation of the cervical proprioceptive system by vibration leads to the displacement of subjective straight visual ahead [14-16].

The vestibular imbalance is readjusted by central compensation, which involves multiple mechanisms, such as adaptation, habituation and substitution. In vestibulopathy patients, proprioception and vision may substitute for parts of the missing vestibular input to sub-serve the insufficient compensation. Strupp, et al. [12] found an increase in muscle spindle input following unilateral vestibular lesion. Therefore, the neck muscle vibration with vestibulopathy patients can affect more displacement of the subjective visual straight ahead than normal subjects.

The stimulus from neck and mastoid can affect both proprioceptive system of neck and vestibular system. On the other hand, the proprioceptive system and vestibular system influence each other. The proprioceptive system in neck is linked to the vestibular system and ocular system for the purpose of maintaining equilibrium and control eye movements [9]. Karnath, et al. [15] reported that when vestibular stimulation (caloric test with ice water) and neck muscle vibration were combined at the same time, their effect was linearly combined either by summation or by cancellation. The SCM is attached to the end of the mastoid process, and vibration in one part may influence the other parts. So, the any part of vibration possibly activates both vestibular and proprioceptive system.

A high correlation was found between the shifts in horizontal eye position and subjective straight-ahead position induced by neck vibration in vestibular neuritis [12]. Another study reported that vibration applied to the head or neck increase the shift of SVV in chronic unilateral vestibular deficits [6]. The authors suggested that sensitivity of the SVH to chronic unilateral vestibular deficits can be improved by applying vibration to the head or neck [6]. Karlberg, et al. [7] reported that vibration-induced shift of the SVH can be explained by the vibration-induced ocular torsion and the magnitude of which is related to the extent of unilateral vertical SCC deficit or otolithic deficit, or both. In our study, shift of SVH due to vibration with IS and CM in compensated stage showed the significant increase of shift of SVH. Similar with these prior reports, our data also suggests the possibility that vibration could enhance the shift of SVH in cVU patients.

To our knowledge, this is the first time that SVH with vibration to the mastoid bone or SCM was performed in aVU group. Unlike with previous study for chronic vestibular deficits, the shift of SVV due to vibration was decreased in aVU. In particular, the shifts of SVH due to vibration at IM were significantly smaller than baseline. The reduction of the vibration-induced shift of SVH with aVU is assumed as a result of ocular torsion in the opposite direction during vibration.

We consider three possible explanations for result that vibration reduce the lesion side shift of SVH in acute stage of unilateral vestibulopathy. First, the vibration at uncompensated stage may activate the remaining lesion side utricular function and evoked torsional shift to healthy side. Second, in the case of vestibulopathy selectively involving superior division, vibration may activate ipsilesional posterior SCC and evoked eye torsional shift to healthy side. Third, the vibration at mastoid and SCM may activate neck proprioceptive system and affected the reducing the lesion side shift. Through this hypothesis, the shift of SVH to affected side can be decreased during vibration. Conversely, in compensative vestibulopathy, shift of SVH due to vibration is increased as previous study reported.

However, there are some limitations of this study. First, we could not demonstrate difference of SVH results according to extents of involved SCC, because we did not assess the SCC function separately. Second, the presence and severity of VIN may have affected the results. In addition, we considered data at different time points in the same patient as separate cases 
and these may have caused error.

In conclusion, the shift of SVH due to vibration in aVU showed the significant decrease of shift of SVH when the vibration is applied on IM, and shift of SVH due to vibration in compensated stage showed the significant increase of shift of $\mathrm{SVH}$ when the vibration is applied on IS and CM.

\section{Acknowledgments}

This study was supported by a grant of the Ministry of Science, ICT and Future Planning grant funded by the Korea government (NRF2012K1A4A3053142).

\section{Conflicts of interest}

The authors have no financial conflicts of interest.

\section{REFERENCES}

1) Friedmann G. The judgement of the visual vertical and horizontal with peripheral and central vestibular lesions. Brain 1970;93:313-28.

2) Böhmer A, Rickenmann J. The subjective visual vertical as a clinical parameter of vestibular function in peripheral vestibular diseases. J Vestib Res 1995;5:35-45.

3) Tabak S, Collewijn H, Boumans LJ. Deviation of the subjective vertical in long-standing unilateral vestibular loss. Acta Otolaryngol 1997;117:1-6.

4) Liu RR, Chen TS, Lin P, Dong H, Lu HH, Zhang N. [Subjective visual horizontal in peripheral unilateral vestibular dysfunction]. Zhonghua Er Bi Yan Hou Tou Jing Wai Ke Za Zhi 2011;46:382-7.

5) Pagarkar W, Bamiou DE, Ridout D, Luxon LM. Subjective visual vertical and horizontal: effect of the preset angle. Arch Otolaryngol Head Neck Surg 2008;134:394-401.

6) Karlberg M, Aw ST, Halmagyi GM, Black RA. Vibration-induced shift of the subjective visual horizontal: a sign of unilateral vestibular deficit. Arch Otolaryngol Head Neck Surg 2002;128:21-7.

7) Karlberg M, Aw ST, Black RA, Todd MJ, MacDougall HG, Halmagyi GM. Vibration-induced ocular torsion and nystagmus after unilateral vestibular deafferentation. Brain 2003;126(Pt 4):956-64.

8) Young ED, Fernández C, Goldberg JM. Responses of squirrel monkey vestibular neurons to audio-frequency sound and head vibration. Acta Otolaryngol 1977;84:352-60.

9) Xie S, Guo J, Wu Z, Qiang D, Huang J, Zheng Y, et al. Vibrationinduced nystagmus in patients with unilateral peripheral vestibular disorders. Indian J Otolaryngol Head Neck Surg 2013;65:333-8.

10) Murofushi T, Curthoys IS. Physiological and anatomical study of click-sensitive primary vestibular afferents in the guinea pig. Acta Otolaryngol 1997;117:66-72.

11) Curthoys IS, Vulovic V, Burgess AM, Cornell ED, Mezey LE, Macdougall $\mathrm{HG}$, et al. The basis for using bone-conducted vibration or air-conducted sound to test otolithic function. Ann N Y Acad Sci 2011;1233:231-41.

12) Strupp M, Arbusow V, Dieterich M, Sautier W, Brandt T. Perceptual and oculomotor effects of neck muscle vibration in vestibular neuritis. Ipsilateral somatosensory substitution of vestibular function. Brain 1998;121(Pt 4):677-85.

13) Hlavacka F, Mergner T, Krizkova M. Control of the body vertical by vestibular and proprioceptive inputs. Brain Res Bull 1996;40:431-4; discussion 434-5.

14) Biguer B, Donaldson IM, Hein A, Jeannerod M. Neck muscle vibration modifies the representation of visual motion and direction in man. Brain 1988;111(Pt 6):1405-24.

15) Karnath HO, Sievering D, Fetter M. The interactive contribution of neck muscle proprioception and vestibular stimulation to subjective "straight ahead" orientation in man. Exp Brain Res 1994;101:140-6.

16) Taylor JL, McCloskey DI. Illusions of head and visual target displacement induced by vibration of neck muscles. Brain 1991;114(Pt 2):755-9. 